\title{
Integrationsorientierte Angebotsstrukturen für Menschen mit Migrationsbiographien/Geflüchtete
}

\section{Eine erwachsenenpädagogische Konzeptualisierung im Spannungsfeld Inter- und Transkultureller Bildung}

\section{Steffi Robak}

Eingegangen: 3. Mai 2018 / Angenommen: 11. Juli 2018 / Online publiziert: 23. Juli 2018

(C) Der/die Autor(en) 2018

Zusammenfassung Der Beitrag systematisiert Perspektiven für die Entwicklung von Angebotsstrukturen für Menschen mit Migrationsbiographien, insbesondere für Geflüchtete. Dafür konzeptualisiert er, theoretisch und empirisch rückgebunden, eine Integrationsvorstellung, die verschiedene bildungsrelevante Prozessebenen und Lerndimensionen aufnimmt. Diese Integrationskonzeptualisierung wird mit dem Bereich der Inter- und Transkulturellen Bildung verschränkt. Auf der Grundlage von Ergebnissen aus Programmanalysen und laufenden Forschungsprojekten wird der Stand der Entwicklung von Angebotsstrukturen für Menschen mit Migrationsbiographie, insbesondere für Geflüchtete reflektiert.

Schlüsselwörter Integration · Angebotsstrukturen für Geflüchtete · Transkulturelle und Interkulturelle Bildung · Programmforschung

\section{Integration-oriented supply structures for people with biographies of migration/refugees}

A conceptualization of adult education in the field of inter- and transcultural education

\begin{abstract}
The article systematizes perspectives for the development of supply structures for people with a migrant background, especially for refugees. For this purpose, the article defines an integration concept that focuses various educationally relevant levels and dimensions, which are entangled with a perspective of the intercultural and transcultural adult and further education. Based on the results from various program
\end{abstract}

Publisher's Note Springer Nature remains neutral with regard to jurisdictional claims in published maps and institutional affiliations.

Prof. Dr. S. Robak $(\bowtie)$

Leibniz Universität Hannover, Hannover, Deutschland

E-Mail: steffi.robak@ifbe.uni-hannover.de 
analyzes studies and ongoing research projects, it furthermore reflects, how these supply structures for people with a migrant background, especially for refugees, are designed.

Keywords Integration - Supply structures for refugees - Transcultural and intercultural education · Program research

\section{Zur Notwendigkeit eines erweiterten Integrationsbegriffs für die Entwicklung von Angebotsstrukturen für Menschen mit Migrationsbiografien/Geflüchtete}

Deutschland ist seit mehreren Jahrzehnten Aufnahmeland für Menschen mit Migrationsbiografien, auch für Geflüchtete. ${ }^{1}$ Es lassen sich Phasen identifizieren, in denen verschiedene Gruppen aus unterschiedlichen Ländern und Milieus Deutschland erreichten (vgl. Pielage et al. 2012; Bundeszentrale für Politische Bildung (bpb) 2017) und für die Bildungsangebote offeriert wurden. Dies geschah mit jeweils nicht-standardisierten Angebotsstrukturen und oblag überwiegend den Kommunen und den regionalen Bildungseinrichtungen. Spätestens 2005 ist mit der Einführung eines Zuwanderungsgesetzes und der Konzipierung von Integrationskursen nicht nur der wichtige Stellenwert von Bildung für das Ankommen in der Gesellschaft platziert worden, sondern es wurden insbesondere mit den Integrationskursen auch systematische Angebote entwickelt. Dies war eine wichtige Wegmarke für die Konzipierung von Angebotsstrukturen für Menschen mit Migrationsbiografien, woran in 2015 für die Entwicklung von Angeboten für Geflüchtete angeschlossen werden konnte. Als Stärke erwiesen sich dabei vor allem die bereits vorliegenden Sprachangebote.

Die politische Anerkennung Deutschlands zunächst als Einwanderungsgesellschaft (Bade 2017) und später als Migrationsgesellschaft (z. B. Mecheril et al. 2010; Heinemann 2014) eröffnete den Blick auf die Rolle der Erwachsenen- und Weiterbildung als eine Transformationsinstanz für gelingende Integration. Nach wie vor ungeklärt ist, was gelingende Integration durch Bildung ausmacht und welche Angebotsstrukturen dafür notwendig sind. Wie ist eine integrative Programmatik zu konzeptualisieren, die bildungswissenschaftliche Aspekte mit Integrationsvorstellungen verbindet, um sie für Angebotsstrukturen und damit das Programmplanungshandeln fruchtbar zu machen (zu Strategien und Wissensinseln des Planungshandelns s. z. B. Gieseke 2008)? Die Erwachsenenbildung/Weiterbildung kann auf keinen entwickelten bildungswissenschaftlichen Integrationsbegriff verweisen. Der Blick zurück auf die verschiedenen Migrationsphasen zeigt jedoch, dass es jeweils zugewiesene Funktionen von Bildung für unterschiedliche Gruppen und Herkunftskulturen gibt. Allen Phasen ist gemeinsam, dass nicht von einem umfänglichen Integrationskonzept über Bildung ausgegangen werden kann, welches berufliche, kulturelle und individuelle Bildungsdimensionen verbindet (Sprung 2009; Robak 2016; Gieseke 2016;

\footnotetext{
1 Der Begriff „Menschen mit Migrationsbiografien“ ist der weiteste Begriff, welcher sowohl die Zugewanderten und Geflüchteten aus verschiedenen zeithistorischen Phasen einbezieht, also auch die verschiedenen Generationen bis hin zu den Geflüchteten, die Deutschland seit 2015 erreichen.
} 
Brandt et al. 2016). Heckmann (2015) stellt fest, dass die in den einzelnen Phasen verfolgten Integrationskonzepte unterschiedlichen Vorstellungen von Fürsorge und Eigenverantwortung folgten. Die Integration der „Gastarbeitenden“ durch Konzepte der sogenannten „Ausländerpädagogik“ war im Kern defizitorientiert und zielte auf eine Assimilation, wohingegen die „Interkulturelle Pädagogik“ auf die Anerkennung der als „fremd“ definierten Migrantinnen und Migranten beruhte (Griese 1984). Mit der Perspektive der natio-ethno-kulturellen Zugehörigkeit wird außerdem auch auf die oftmals unkritische Aufeinanderbezogenheit der Konzepte Nationalität, Staatsangehörigkeit und Ethnie im Rahmen der Migrationspolitik hingewiesen (Mecheril 2004). Präsent bleibt bis dato der Anspruch einer umfassenden Assimilation bis hin zur Aufgabe der eigenen Identität, besonders wenn es etwa um religiöse Identität geht (vgl. Hofheinz 2016). Daran schließt sich eine Skepsis, ob Integration im Sinne von Assimilation überhaupt möglich ist, da sie genau den Kern der Identitätsbildung berührt. Die dahinter stehende leitende mehrheitsgesellschaftliche Perspektive ist die Frage der Eingleichung von Differenzen einerseits und die Sorge um Statuseinbußen im eigenen Nahbereich andererseits. Auf Letzteres verweisen die Studien von Zick und Klein (2014) sowie Krause et al. (2016), wenn etwa Ängste, Etabliertenvorrechte einzubüßen, thematisiert werden (ebd., S. 160). Diese und andere Ergebnisse verweisen darauf, dass für die Realisierung einer Migrationsgesellschaft unter Integrationsgesichtspunkten sowohl die Menschen mit Migrationsbiografien als auch die Mehrheitsgesellschaft durch Bildung $\mathrm{zu}$ adressieren sind. $\mathrm{Zu} \mathrm{Recht}$ weist Öztürk (2014) darauf hin, dass für empirische Forschungen auch die verschiedenen Generationen berücksichtigt werden müssen (vgl. Öztürk 2014, S. 49). Diese mehrperspektivischen Forschungsergebnisse bilden eine Grundlage für die Angebotsstrukturen, die sich an Menschen mit Migrationsbiografien, und hier aufgrund der Integrationskurse insbesondere an die Geflüchteten seit 2015, richten.

Insgesamt ist noch von einem zu geringen Differenzierungsgrad an Wissensstrukturen über die verschiedenen Gruppen mit Migrationsbiografien auszugehen. Es kann bereits auf Forschungen über Migration nach Deutschland verwiesen werden, etwa über Lebenslagen und Ressourcen junger Frauen und Mädchen und besondere Kompetenzen der Zweisprachigkeit (Boos-Nünning und Karakaşoğlu 2005), über individuelle Faktoren der Bildungsteilhabe (Öztürk 2012, 2014), individuelle und gesellschaftlich-strukturelle Faktoren der Weiterbildungsteilhabe von Frauen mit Migrationshintergrund (Heinemann 2014), über Hochqualifizierte sowie auch Transmigranten (Hamburger et al. 2005; King und Koller 2006; Sievers et al. 2010). Über die Geflüchteten, die seit 2015 Europa und Deutschland erreichen, liegen bis dato nur in sehr begrenztem Umfang Wissensressourcen vor, die als Grundlage für eine integrationsförderliche Ausgestaltung von Bildungsprozessen dienen können (Böhmer 2016). Einerseits finden sich theoretische konzeptionelle Auseinandersetzungen und reflexive Ausarbeitungen über die Bildungsarbeit mit Geflüchteten (z. B. Ziese und Gritschke 2016; Sievers und Grawan 2018; Schiffauer et al. 2017), andererseits liegen erste Erkenntnisse über die Bildungshintergründe der Geflüchteten vor: Die ersten Ergebnisse einer repräsentativen Längsschnittbefragung von 4527 Erwachsenen und ihren 5438 Kindern zeigt, dass $40 \%$ der erwachsenen Geflüchteten mit gültigen Bildungsangaben (2013 bis Januar 2016) eine weiterführende Schule besucht haben $-35 \%$ haben dort einen entsprechenden Schulabschluss er- 
worben - $12 \%$ verfügen über Bildung ,auf Grundschulniveau“. Weitere $13 \%$ haben keine Schule besucht, $11 \%$ besitzen einen Abschluss für den tertiären Bereich (ISCED 6, 7 oder 8; Hochschule, Meister, Techniker etc.). 61\% der Geflüchteten (alle Schulformen) haben bei ihrer Ankunft in Deutschland einen Schulabschluss; 16\% besitzen einen Hochschul- oder beruflichen Abschluss. Zielvorstellungen: $47 \%$ der Geflüchteten haben die Absicht, in Deutschland einen Schulabschluss zu machen. Ein noch größerer Teil, nämlich etwa zwei Drittel der Geflüchteten streben einen beruflichen Abschluss an (BMBF 2018, vom Institut für Arbeitsmarkt- und Berufsforschung (IAB) durchgeführt). An diesen Ergebnissen wird deutlich, dass das Weiterbildungssystem auf allen Ebenen und für alle Bildungsbereiche herausgefordert ist, Angebote zu entrichten. Dass für eine gesellschaftliche Integration als solche besonders der umfassende Zuwachs an kulturellem Kapital förderlich ist, ist im Bewusstsein angekommen (Heckmann 2015). Dieses wird vor allem in Bildungsprozessen erworben (z. B. Sprachkurse, Zertifikate, Schul-/Berufsabschlüsse) (ebd.; Heinemann 2014; Sprung 2009; Öztürk 2014).

Besonders wichtig ist der Hinweis von Heckmann (ebd.), dass eine ethnische Schichtung vermieden werden muss, die dann wiederum mit dem Begriff der „Parallelgesellschaft“ besetzt werden kann. Hier ist der Bildungsbereich gefordert, denn: „Wenn Bildung nicht möglich ist oder verhindert wird, erfolgt früh eine Einmündung in Beschäftigung oder es wird eine Funktion in ethnischen Gemeinden übernommen“ (ebd., S. 4). Betrachten wir Integrationsbegriffe, auf die im Bildungskontext verwiesen wird, wird deutlich, dass die Integrationsverständnisse zwei Verkürzungen aufweisen: Sie beziehen sich zum einen ausschließlich auf Menschen mit Migrationsbiografien, die ja grundsätzlich auch eine hohe Heterogenität aufweisen, und sie nehmen die Mehrheitsgesellschaft zumeist nicht in den Blick. Zum anderen erfassen sie auch bei der Adressierung der Zugewanderten nicht umfassend die Bildungsherausforderungen für alle relevanten Bereiche der Partizipation und Gestaltung der Lebenswelten. Für Bildungsfragen anschlussfähig ist etwa die Unterscheidung von Esser (2001) in Systemintegration und Sozialintegration. ${ }^{2}$ Die Entwicklung von Angebotsstrukturen an Geflüchtete muss die darin benannten Anforderungen im Blick haben. Jedoch konnten diese Forderungen, so belegen Bildungsmonitoringstudien, bereits für die hier lebenden Gruppen mit Migrationsbiografien vor 2015 nicht konsequent realisiert werden. Sie sind häufiger von einer Kumulation benachteiligender Faktoren betroffen (Öztürk 2016). Hinzu kommt, dass Aspekte der Diversität nicht berücksichtigt werden. Das heißt, meist wird der natio-ethno-kulturelle Hintergrund der Zugewanderten (zum Begriff s. Mecheril 2004) besonders als Differenzmarker konstruiert. Zu berücksichtigen sind aber Durchdringungen und Wechselwirkungen mit den Aspekten Geschlecht, Alter, Religion, Bildungshintergrund, Milieu, Habitus etc. (s. dazu die Ausarbeitungen in Hauenschild et al. 2013; Robak 2013).

\footnotetext{
2 Die Sozialintegration umfasst die Bereiche Kulturation (Erwerb von Wissen, Fertigkeiten und Sprache), Platzierung (Übernahme von Positionen und die Verleihung von Rechten), Interaktion (Aufnahme sozialer Beziehungen im alltäglichen Bereich) und Identifikation (emotionale Zuwendung zu dem betreffenden sozialen System). Für die Systemintegration ist eine konsequente Aufnahme im Bildungssystem und in der Arbeitswelt bedeutsam (Esser 2001).
} 
Der vorliegende Artikel befasst sich mit den folgenden Leitfragen: Wie kann der Blick auf Integration geweitet werden und welche Perspektiven und Bildungsdimensionen sind dafür zu berücksichtigen? Welche Wissensstrukturen über Menschen mit Migrationsbiografien können herangezogen werden? Wie können die zentralen Lebensbereiche und die demokratischen Prämissen orientierend eingebunden werden? Gibt es darüber ein geteiltes Grundverständnis, etwa wenn wir auf kulturelle Unterschiede unter verschiedenen Aspekten schauen? ${ }^{3}$

Im nächsten Kap. 2 wird eine Konzeptualisierung für gelingende Integration beschrieben. Diese basiert auf empirischen und theoretischen Ergebnissen, die im Rahmen meiner Habilitationsstudie (Robak 2012) ${ }^{4}$ sowie weiterer (eigener) Forschungsergebnisse generiert wurden (Robak und Petter 2014; Zimmer et al. 2015; Fleige et al. 2015; Robak und Grawan 2016). ${ }^{5}$ Im Artikel wird außerdem argumentiert, dass die in Kap. 2 dargelegten Prozessebenen und Lerndimensionen für die Angebotsentwicklungen für Menschen mit Migrationsbiografien, insbesondere für die seit 2015 nach Deutschland Geflüchteten zu berücksichtigen sind bzw. eine orientierende Funktion einnehmen können. Die dafür relevanten Bildungsbereiche werden in Kap. 3 unter den Begriffen der Interkulturellen und Transkulturellen Bildung entwickelt. Programmanalysen aus den Jahren 2012 und 2017 werden dann in Kap. 4 als Zugang herangezogen, um die Themenstrukturen und Bildungszugänge der Interund Transkulturellen Bildung in ihrer Ausdifferenzierung darzulegen und daraufhin zu betrachten, inwiefern sich die Prozessebenen der Integrationskonzeptualisierung darin wiederfinden. Inter- und Transkulturelle Bildungszugänge durchziehen alle relevanten Fachbereiche und werden für die Konzeptualisierung von Integrationsprozessen als zentral betrachtet. Der Vergleich der Programmanalysen zwischen den Jahren 2012 und 2017 soll aufzeigen, wie die Bildungsbedarfe und -bedürfnisse der Geflüchteten, die seit 2015 Deutschland erreichen, einbezogen werden und ob sich ein Integrationskonzept entwickelt, das die dargelegten Prozessebenen einbezieht. Da sich in den letzten Jahren die Unternehmen ebenfalls im Bereich der beruflichen Integration engagieren, aber nicht von systematischen Programmstrukturen für berufliche Integration der Geflüchteten in Unternehmen ausgegangen werden kann, wird in Kap. 5 am Beispiel exemplarischer Projektanalysen hinterfragt, welche Vorstellungen der Verknüpfung von Bildung und Integration für die Prozessebene II, Professionalisierung und Qualifizierung (Kap. 2) vorzufinden sind. Diese Prozess-

\footnotetext{
3 Vgl. hierzu das Themenheft „Integration“ in Hessische Blätter für Volksbildung, 4, (2016).

${ }^{4}$ In der Habilitationsstudie wurde untersucht, wie deutschsprachige Expatriates, die für unterschiedliche Zeiträume, aber mindestens ein Jahr nach China entsendet werden, sich dort vor allem beruflich, aber auch in der privaten Lebenswelt integrieren. Dafür wurde eine bildungswissenschaftliche Perspektive eingenommen, die mit einer kulturwissenschaftlich-soziologischen Theoretisierung verbunden wurde. Diese fragte danach, auf welche Wissensstrukturen einerseits zurückgegriffen und welche Wissensstrukturen andererseits generiert wurden, um Hybridbildungen (nach Reckwitz 2006), im Sinne von neu geformten (Arbeits-)praktiken so vorzunehmen, dass die entsendeten Führungskräfte die größtmöglichen Handlungsund Gestaltungsspielräume, sowohl beruflich als auch in allen anderen Lebenszusammenhängen entwickeln können.

5 Laufende Forschungsprojekte: „Cultural Heritage als Ressource? Konkurrierende Konstruktionen, strategische Nutzungen und multiple Aneignungen kulturellen Erbes im 21. Jahrhundert (CHER)“ und „Facilitating Migration and Workplace Integration with Qualified Personnel and Concepts (MaWIC)“.
} 
ebene müsste insbesondere neu bzw. weiterentwickelt werden, da die berufliche Integration auch (bildungs-)politisch gewünscht ist und forciert wurde.

\section{Bildungsrelevante Konzeptualisierung von Integration als Perspektive für Angebotsentwicklungen}

Betrachten wir Diskurse und Akzentsetzungen in der Angebotsentwicklung für Integration, ist zunächst auf die Rolle von Sprachkursen hinzuweisen. Angebote zum Spracherwerb sind für gelingende Integration durch Bildung von zentraler Bedeutung. Das Integrationsgesetz hat insofern einen wichtigen strukturellen Anstoß gegeben, als bereits viele Erfahrungen mit Integrations- und Sprachkursen vorliegen. Die Integrationskurse können, rein quantitativ betrachtet, durchaus als Erfolgsgeschichte interpretiert werden. So wurden bereits im Jahr 2015283.404 Teilnahmeberechtigungen ausgestellt. Davon haben ca. 179.400 einen Integrationskurs begonnen. $60 \%$ von diesen Personen erreichten dabei Niveaustufe B1 (32\% Stufe A2) in der deutschen Sprache. Die Volkshochschulen hatten in 2015 einen Gesamtträgeranteil von 36\% (BAMF 2015). Eine Interviewstudie mit Teilnehmerinnen und Teilnehmern in Bremen und Bremerhaven zeigt: Integrationskurse, Sprachkurse und thematisch ausgerichtete Zielgruppenangebote für Menschen mit Migrationsbiografien stellen einen gleichbleibend bedeutsamen Zugang zum Weiterbildungsangebot dar und werden auch so genutzt. Sie werden darüber hinaus von den Adressatinnen und Adressaten sehr positiv bewertet (Zimmer et al. 2015). Vorarbeiten von Zimmer (2013) hatten darauf verwiesen, dass Teilnehmende an Integrationskursen diese als einen wichtigen Schritt für ihr Ankommen in Deutschland bewerten. So gelingt es häufig, aus den Integrationskursen heraus eine Brücke in das Regelangebot der Weiterbildung zu schlagen (Zimmer et al. 2015). Sprachkurse allein reichen jedoch nicht aus; Kurse, die eine gelingende Integration zum Ziel haben, brauchen Konzepte in Bezug auf Arbeit, Beruflichkeit und Teilhabe in sozialer, politischer und kultureller Hinsicht. Die Berufliche Bildung ist ebenso gefordert wie die Politische, Personale und Kulturelle Bildung (vgl. Heinemann und Robak 2012). Besonders die Kulturelle Bildung erweist sich als wichtiges Zugangsportal - v. a. Malen, Musik und Tanz -, aber auch die Gesundheitsbildung. Weitere Angebote, wie Fremdsprachen und Anpassungsqualifizierungen im beruflichen Bereich, haben ebenfalls einen hohen Stellenwert (Zimmer et al. 2015).

Blicken wir aus dieser Bildungsperspektive auf Integration in Bezug auf die Gruppen mit Migrationsbiografien, hier insbesondere Menschen im Asylbewilligungsprozess, so sind, wenn wir die oben genannten Forschungsergebnisse auswerten, drei Prozessebenen und drei Lerndimensionen für Bildungsprozesse mit dem Ziel der gelingenden Integration einzubeziehen (Abb. 1). Das nachfolgende Modell wurde abduktiv entwickelt, d.h. es basiert auf einem rekursiven Forschungsprozess, der theoretische und empirische Aspekte konzeptualisiert, empirisch analysiert und wie- 


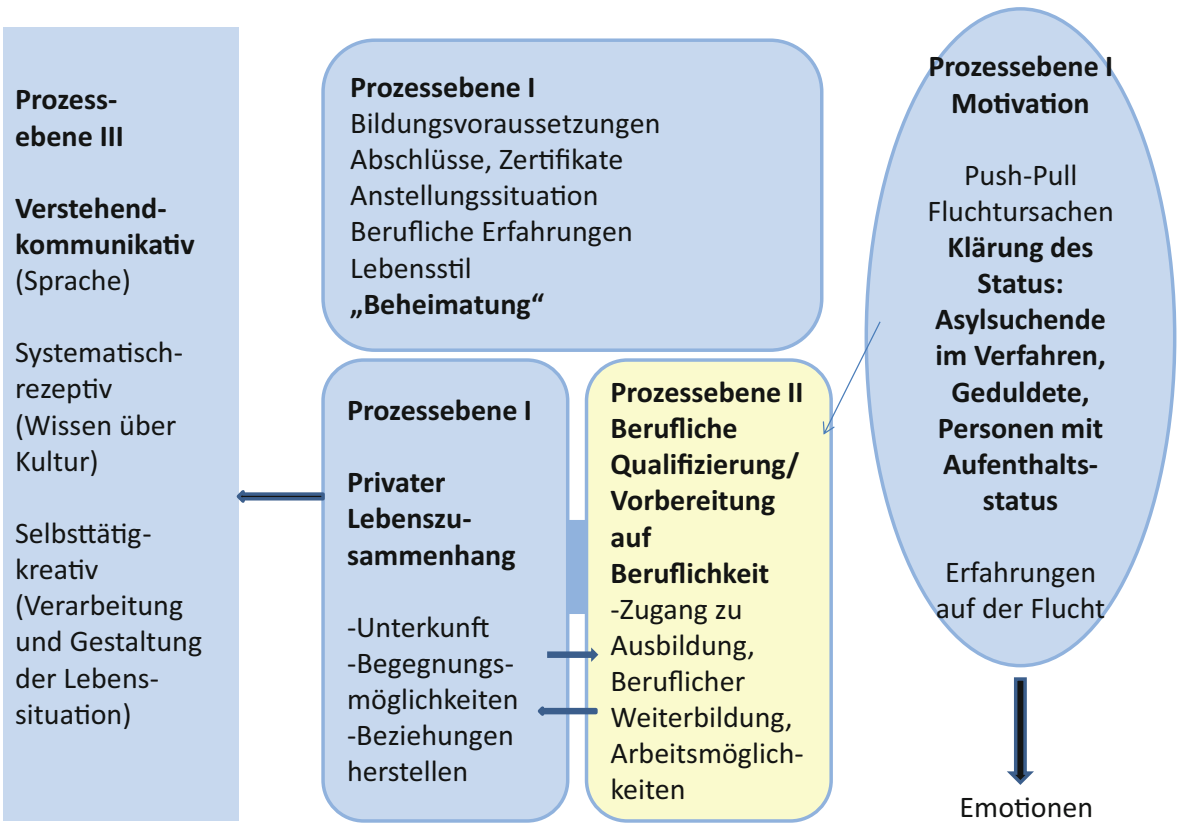

Abb. 1 Prozessebenen der Integration und Wirkungszusammenhänge (eigene Darstellung)

derum theoretisiert (Reichertz 2003; Robak 2012, Kap. 5). ${ }^{6}$ Nach der Nennung der empirisch gewonnenen Prozessebenen und Lerndimensionen werden für die folgenden Betrachtungen Zusammenhänge für Menschen mit Migrationsbiografien, insbesondere Geflüchtete, die Deutschland jüngst erreichten, spezifiziert. Darin fließen zusätzlich Erkenntnisse aus den in Kap. 1 bereits genannten Studien und Analysen ein (Robak 2012; Robak und Petter 2014; Zimmer et al. 2015; Fleige et al. 2015; Robak und Grawan 2016):

- Prozessebene I, psychodynamische Akkulturation/Anpassung - individuelle biografische Bedingungen und Lernvoraussetzungen

- Prozessebene II, Professionalisierung und Qualifizierung - individuelle Lernformen

\footnotetext{
${ }^{6}$ Das empirische Sample der Habilitationsstudie umfasste 57 Interviews mit 52 Personen, die überwiegend als Expatriates für unterschiedliche Zeiträume in China arbeiten. Es wurde ein spezifischer abduktiver Forschungsprozess entwickelt, in dem sukzessive theoretische interdisziplinäre Wissensstrukturen ausgewertet und in die Leitfadenerstellung problemzentrierter und zugleich hochstrukturierter Interviews eingebunden wurden. Gleichzeitig erfolgte eine Kategorienweiterentwicklung auf der Grundlage der geführten Interviews, die ebenfalls in die Leitfadenerstellung einflossen. In einem rekursiven Prozess wurde ein Interview- und Auswertungsraster entwickelt, welches sowohl individuell berufsbiografische Aspekte als auch berufsgruppenspezifische Fragen, Fragen zum Akkulturationsprozess, zur Aneignung von Kultur und kultureller Differenz, zur Rolle von Unternehmenskulturen und den Anforderungen von Zusammenarbeit in globalen Unternehmen einbezog. Alle Interviews wurden dann unter Nutzung des entwickelten Rasters einer erneuten Analyse unterzogen. Die Auswertung erfolgte inhaltsanalytisch und folgte den hier dargelegten Prozessebenen und Lerndimensionen.
} 
- Prozessebene III, Bildung und Kulturalität - individuelle Zugänge zum kulturellen Lernen und zu kultureller Bildung

Die gewonnenen Lerndimensionen sind:

- Lernformen/Arbeitspraktiken

- kulturelles Wissen/Kulturstandards

- Deutungsmuster

Prozessebene I: Psychodynamische Akkulturation/Anpassung/Gestaltung des Lebenszusammenhangs - individuelle biografische Bedingungen und Lernvoraussetzungen: Prozessebene I greift abduktiv auf Erkenntnisse der Akkulturationsforschung (z.B. Berry 2005; Koop und Schäfers 2006), der Migrationsforschung (z. B. Mecheril 2003), der Biografieforschung (z. B. Alheit 1995; Alheit und Dausien 2002) und auf grundlagentheoretisches erwachsenenpädagogisches Wissen über Lernen im Lebenszusammenhang (z.B. Friebel et al. 2000, 2008; Gieseke 2016) zurück. Prozessebene I umfasst die Konstellationen, inklusive der Bildungskonstellationen, die Einfluss auf die Möglichkeiten des Ankommens, der „Beheimatung“ in der privaten und beruflichen Lebenswelt nehmen. Das Ankommen im Aufnahmeland nimmt die ganze Person in Anspruch: Die Gestaltung der Lebenswelt, die Entwicklung eines annehmbaren Lebensstils, der Anknüpfungspunkte an Praktiken der Herkunftskultur zulässt und gleichzeitig das Neue aufnimmt. Die gesamte Lebensplanung muss unter Umständen in der Schwebe gehalten werden, wenn der Aufenthaltsstatus ungeklärt ist oder die Rückkehr- bzw. Bleibeperspektiven ungeklärt sind. Wenn wir auf Geflüchtete aus Krisen- und Kriegsgebieten schauen, kann man nicht mit denselben Bedeutungen von Biografisierungsprozessen sprechen, wie sie für andere Gruppen in Deutschland analysiert wurden und relevant sind (vgl. Alheit 1995). Es geht natürlich auch um Zukunftsperspektiven, um biografische Sinngebung, aber auch um die Verarbeitung von Todesängsten und Traumata (z. B. Zimmermann 2012) verschiedenster Art. In welcher Form eine Akkulturation möglich ist, hängt auch vom Aufenthaltsstatus ab und von der Motivation, die mit dem Verbleib in Deutschland aufgebaut werden kann.

Betrachten wir das Schema, so haben die Migrations- bzw. Fluchtursachen, die Motivation, der Status und die zuvor bereits genannten Fluchterfahrungen einen Einfluss auf die Interessen der Integration (s. rechts im Schema). Die Bildungsvoraussetzungen, die erworbenen Abschlüsse, ggf. die Anstellungssituation und die beruflichen Möglichkeiten und Erfahrungen (s. oben im Schema) beeinflussen zudem die Ausformung von Interaktionsmöglichkeiten und damit die Gestaltung der Lebenswelt und der Beziehungen (Gieseke 2016) darin. Die Gestaltungsmöglichkeiten, die mit den vorhandenen Konstellationen im privaten Lebenszusammenhang mit ihren Optionen der Begegnung und des Beziehungsaufbaus gegeben sind oder geschaffen werden, nehmen wiederum Einfluss auf Prozessebene II, die die Möglichkeiten der beruflichen Qualifizierung bzw. der Vorbereitung auf Beruflichkeit beschreibt.

Angebote, die Prozessebene I adressieren, bearbeiten biografische Erlebnisse und Erfahrungen sowie neue Erfahrungen in der ankommenden Kultur, ermöglichen unabhängig vom Bleibestatus Partizipation und Gemeinschaft, unterstützen die Gestal- 
tung der Lebenswelt, reflektieren und unterstützen die Ausformung neuer Zugehörigkeiten, auch indem Optionen der Beziehungsaufnahme zur Mehrheitsgesellschaft offeriert werden. Dies geschieht nicht nur über Bildungsangebote, sondern auch über ein breites Spektrum an zivilgesellschaftlichem Engagement (s. dazu die Dokumentationen in Schiffauer et al. 2017).

\section{Prozessebene II: Professionalisierung und Qualifizierung - individuelle Lernfor-}

men: Prozessebene II rekurriert auf Grundlagenwissen über Formen und Funktionen der beruflichen, inklusive der arbeitsplatzbezogenen Weiterbildung, Lernkulturen und Lernformen (z. B. Baethge und Baethge-Kinsky 2004; Schiersmann 2006; Dehnbostel 2008). Der Aufbau einer Beruflichkeit (vgl. Kraus 2006), das Erlernen oder auch die Anerkennung eines Berufs, die Nachqualifizierung bzw. die Anleitung oder das Coaching am Arbeitsplatz mit der Möglichkeit, einen Beruf auszuüben, ist eine grundsätzliche Prozessebene, die mit Bildung zu adressieren ist. Dazu gehören Möglichkeiten der Qualifizierung auf verschiedenen Niveaus. Der Beruf, berufliche Tätigkeiten und die Möglichkeiten der Qualifizierung darin formen prägende Interaktionszonen aus, die entscheidend für Integration sind. Wie schwer es ist, eine Bildungskette für die Geflüchteten aufzubauen, die in berufliche Bildung und Weiterbildung einmündet bzw. damit verbunden wird, wurde in den vergangenen drei Jahren viel diskutiert. Die Europäischer Sozialfonds (ESF)-Förderlinien erwiesen sich hier als entscheidend, um Qualifizierungsprojekte für eine berufliche Integration zu realisieren (Robak 2015). Die Stellung der Erwachsenen- und Weiterbildung, etwa als intermediäre Institution, die auch die Systemintegration mit absichert, wird unterschiedlich gesehen. Wichtig ist es, vor dem Hintergrund der aktuellen Anforderungen und Diskurse auf die notwendige Koppelung von Qualifikationsanalyse, Beratung und Begleitung hinzuweisen. Die individuelle berufliche Weiterbildung ist zu stärken, da die betriebliche Weiterbildung nur einen Bruchteil der Menschen erreicht und zudem selektiv strukturiert ist.

Angebote, die die Prozessebene II adressieren, liegen im breiten Feld der beruflichen Bildung und Weiterbildung bis hin zum arbeitsplatzbezogenen Lernen. Sie bereiten sowohl auf fachliche als auch überfachliche Kompetenzentwicklung vor und können sowohl abschlussbezogen mit Zertifikaten als auch ohne strukturiert sein. Sie sollten die langfristige Beruflichkeit als auch den unmittelbaren Tätigkeitsbezug perspektivisch einbeziehen.

Prozessebene III: Bildung und Kulturalität - individuelle Zugänge zum kulturellen Lernen und zu Kultureller Bildung: Diese Prozessebene fokussiert auf die kulturelle Beschäftigung mit dem Aufnahmeland und die eigene Positionierung und Beheimatung darin. Der Einzelne wird als kulturgestaltendes Individuum über ver- 
schiedene Partizipationstore ${ }^{7}$ des Lernens dazu angeregt und befähigt, sich diesem zu nähern, darin zu positionieren, sich selbst dazu zu verhalten und letztlich Kultur mitzugestalten. Dies fördert die Ausformung von Kulturalität. In die Konzeptualisierung dieser Prozessebene fließen erwachsenenpädagogische und interdisziplinäre Kenntnisse über kulturelle Bildungsprozesse (Gieseke et al. 2005; Fleige et al. 2015), Inter- und Transkulturelle Bildung (Robak 2012; Robak und Petter 2014), über Prozesse der Kulturformung (Scharfe 2002; Bhabha 2000), individuelle Kulturalität ${ }^{8}$ (Göller 2000) und Personalisation, den Umgang mit Fremdheit (Schäffter 1991) sowie gesellschaftliche Entwicklungen und transformatorische Prozesse in der Postmoderne ein (Reckwitz 2006). Für die Kulturelle Bildung liegt eine theoretisch und empirisch abgesicherte Systematik vor (Gieseke et al. 2005; Fleige et al. 2015), auf die zurückgegriffen werden kann. Eine systematisch-rezeptive Beschäftigung bedeutet für gelingende Integration eine aktive Auseinandersetzung mit dem Aufnahmeland, seiner Historie, seinen Artefakten, der Region, seinen kulturellen und gesellschaftlichen Entwicklungen und der gesellschaftspolitischen Situation. Dies kann Hochkultur ebenso einschließen wie Populärkultur. Hinzugenommen wird hier auch Sprache als verstehend-kommunikativer Zugang, da die Aneignung von Sprache eine kulturelle Auseinandersetzung in sich trägt. Ein selbsttätig-kreativer Zugang könnte etwa das Erlernen eines Instruments, das Theaterspielen oder das Erlernen eines Tanzes sein.

Bildung und Kulturalität sind in ihren Zugängen zu Bildungsangeboten im Zusammenhang zu sehen. Die Ausformung von (Mehrfach-)Zugehörigkeiten kann als ein zentraler Aspekt für Beheimatung und gelingende Integration angesehen werden (nach Mecheril 2004; Robak und Grawan 2016). Um die Individuen in ihren Erfahrungen, Deutungen und Wissensstrukturen anzusprechen und diese aufzugreifen und dabei gleichzeitig Potenziale der Kreativität zu aktivieren, braucht es eine umfassende Konzeption Kultureller Bildung, Interkultureller Bildung und Transkultureller Bildung. Diese finden sich in dem oben dargestellten Schema auf der linken Seite mit den Partizipationsportalen verstehend-kommunikativ, systematisch-rezeptiv und selbsttätig-kreativ. Was dies alles umfasst, wurde an anderer Stelle ausführlich entwickelt (vgl. Robak 2012; Fleige et al. 2015).

\footnotetext{
${ }^{7}$ Partizipationsportale sind beschreitbare differenzierte Zugangswege zu Kultur und Bildung (Gieseke et al. 2005). Die Portale sind mit Wissensformen, Aneignungswegen und Lernorten verbunden (ebd.): organisierte Lernangebote (didaktisch strukturierte Angebote in Erwachsenenbildungseinrichtungen); beigeordnete Bildung (Angebote in Nicht-Erwachsenenbildungseinrichtungen wie Museen und soziokulturellen Zentren); kulturelle Praxis bzw. Sparte (z. B. Chor); kulturelles Erlebnis bzw. Event (z. B. Kulturfeste) (ebd.; Robak und Fleige 2012). Portale liegen quer zu Fachbereichen und beziehen Intentionen von Veranstaltungsformen und Lernformen mit ein.

${ }^{8}$ Kulturalität entsteht durch kulturelle Gestaltung und die Fähigkeit, Arten der kulturellen Gestaltung zu thematisieren. Sie realisiert sich durch Sinnformierungen, Teilhabe, Formen der Selbstbeziehung und des reflexiven Rückbezugs auf kulturelle Gemeinschaften, um auf der Grundlage gestaltender Teilhabe neuen Sinn zu ermöglichen (Göller 2000). „Mit Kulturalität ist die spezifische Sinnbestimmtheit des Menschen gemeint; (menschliche) Kultur ist bzw. (menschliche) Kulturen sind durch diese charakterisiert (...). Diese Sinnbestimmtheit impliziert die aktiv-spontane Selbstbeziehung des Menschen auf sich selbst, auf Mitmenschen, menschliche Handlungen, Äußerungen, Erzeugnisse, Produkte, auf die Welt oder Weltstücke“ (ebd., S. 272).
} 
Gelingende Integration, die in Bildungszusammenhängen an der Strukturierung von Bildung und am Individuum ansetzt, berücksichtigt mindestens drei Lerndimensionen, auf denen Lern- und Bildungsprozesse stattfinden. In allen oben genannten Prozessebenen spielen die im Folgenden angesprochenen Dimensionen des Lernens und der Bildung eine Rolle. Es ergibt sich also ein mehrdimensionales Modell für Integrationsprozesse aus der Erwachsenen- und Weiterbildungsperspektive, das orientierend für die Entwicklung von Angebotsstrukturen sein kann.

Lerndimension I: Lernformen und Arbeitspraktiken (im Arbeitszusammenhang), die so angelegt sind, dass die Individuen für sich Lern-, Bildungs-, Qualifizierungsund im umfassenden Sinne Aneignungsprozesse realisieren können. Im beruflichen Kontext kann dies bedeuten, dass eine lernende Aneignung im Prozess, z. B. mit Formen der Unterweisung oder aber gekoppelt an Qualifizierung in unterschiedlichen Lernformen, stattfindet. Hier ist die berufliche Weiterbildung angesprochen, denn eine grundständige berufliche Ausbildung ist für viele der Geflüchteten notwendig und von diesen auch erwünscht. Dies ist eine wichtige Voraussetzung, reicht aber für eine gelingende Integration noch nicht aus. Wenn eine Partizipation an Gesellschaft realisiert werden soll, dann braucht es den Aufbau von Deutungen und das Verstehen von Deutungsmustern, die in einer Gesellschaft höchst heterogen und gleichzeitig kulturell geprägt sind.

Lerndimension II: Erfahrungen und Deutungen sind konstitutiv für die Handlungs- und Gestaltungsfähigkeit in der neuen Gesellschaft (Dybowski und Thomssen 1976; Arnold 1985; Schüssler 2000; Gieseke 2010). Diese Aspekte sind so einzubeziehen, dass sowohl Anschlüsse an Bisheriges realisiert als auch neue Erfahrungen und Deutungen aufgebaut werden können. Für die Ausformung neuer Zugehörigkeiten und die gesellschaftliche Platzierung ist die Möglichkeit, Erfahrungen zu machen und neue Deutungen aufzubauen, besonders konstitutiv.

Lerndimension III: Kulturelles Wissen (in Anlehnung an Kulturstandards nach Alexander Thomas, s. ausführlich Robak 2012), so dass historisch wirksame Wissensressourcen, die in Interaktionskontexten in Milieus ganz unterschiedlich wirksam sind, verstanden und einbezogen werden können.

Für solch einen angelegten Integrationsbegriff, der bis hierher auch nur die Gruppen mit Migrationsbiografie berücksichtigt, braucht es eine Weiterentwicklung von Konzepten, einen Verständigungsprozess, der Interkultur - im bisherigen Verständnis des Verstehens kultureller Differenzen - und Transkultur im Sinne gemeinsamer Kulturgestaltung einbezieht und einen konsequenten Ausbau von Fördermöglichkeiten für Bildungsangebote in den verschiedenen Themenbereichen und Zugangsportalen. Eine transkulturelle Haltung einzunehmen bedeutet in Anlehnung an Welsch (2005) und Reckwitz (2003, 2006), dass alle an einer Gesellschaft Partizipierenden kulturformend wirken, indem sie Praktiken einbringen, die den jeweiligen Milieus und natio-ethno-kulturellen Hintergründen entspringen (vgl. Robak 2012). Die Ausformung von (Mehrfach-)Zugehörigkeiten betrachten wir als einen Zugang, an der Kulturformung von Gesellschaft zu partizipieren (Robak und Grawan 2016). Dies wird über Bildung befördert. Diese drei Lerndimensionen bilden, neben einer transkulturellen Orientierung, die Grundlage zur Entwicklung integrationsförderlicher Angebotsstrukturen, auf die im folgenden Kap. 3 fokussiert wird. 


\section{Inter- und Transkulturelle Bildung als Rahmen der Entwicklung integrationsförderlicher Angebotsstrukturen}

Angebotsstrukturen für Menschen mit Migrationsbiografien, auch wenn sie die jüngst Geflüchteten adressieren, können sich an den oben dargestellten Prozessebenen und Lerndimensionen orientieren, wenn potenziell davon ausgegangen wird, dass sie Teil der Migrationsgesellschaft in Deutschland werden sollen. Diesem mehrdimensionalen Integrationskonzept entspricht ein Bildungsbegriff, der die oben genannte transkulturelle Haltung der Kulturgestaltung unterstützt und davon ausgeht, dass sich die Gesellschaft in einem Prozess der Transformation befindet, in dem Migrationsbewegungen beeinflussender Teil von Hybridisierungsprozessen werden, die die Gesellschaft nachhaltig beeinflussen und verändern werden (Bhabha 2000; Reckwitz 2006). Interkulturelle und Transkulturelle Bildung sind, wie bereits in Kap. 1 eingeführt, die Bildungszugänge, die in allen relevanten Fachbereichen vorkommen und speziell den Zusammenhang von Bildung, kultureller Differenz, Umgang mit Fremdheit thematisieren und dabei Integration mitdenken.

Es wird aufgrund der gesellschaftlichen Entwicklungen, der sichtbar werdenden Lernbedürfnisse und Lerninteressen davon ausgegangen, dass sowohl eine Interkulturelle Bildung als auch eine Transkulturelle Bildung weiter auszudifferenzieren sind und beide Auslegungen als Teil Kultureller Bildung und zugleich in Übergängen zu anderen Bildungsbereichen, wie etwa Politische und Berufliche Bildung auszuarbeiten und zu offerieren sind.

Das heißt, wir folgen der Prämisse, dass für gelingende Integration beide Begriffe relevant sind und unterschiedliche Angebotsstrukturen benötigt werden, diese aber eine Gesamtkonzeption ausmachen. Diese Konzeptualisierung ermöglicht die Realisierung von Programmen und Konzepten, die den Spannungsbogen der Bearbeitung aller drei Prozessebenen und Lerndimensionen adressiert, dabei aber auch der Frage nachgeht, wie gemeinsame Gestaltungsprozesse, die im weiten Sinne Kulturformungen unterstützen, über Bildung adressiert werden können. Die Begriffe „Interkulturelle“ und „Transkulturelle Bildung“ sind bereits in verschiedenen Veröffentlichungen dargelegt worden (vgl. jüngst in Fleige et al. 2015; Robak und Grawan 2016; Robak 2017). Im Bereich Interkultureller Bildung werden Angebote kommunikativer Verständigung, identitärer Selbstvergewisserung bzw. Identitätsentwicklung, der Dekonstruktion von Kulturalisierungen (Kaschuba 1995) sowie des Verstehens und Erlernens kultureller Praktiken (Robak 2012) konzeptioniert. Durch die Teilhabe an Kultur wird Ambiguitätstoleranz und Empathie erlernt, indem die eigene Empfindungsfähigkeit, gekoppelt an das Verstehen der Praktiken anderer Kulturen, entwickelt wird. Unter diesem Kulturbegriff werden auch Themen wie Diskriminierung, Rassismus und Antisemitismus bis hin zum Umgang mit Folgewirkungen von Kolonialisierung platziert. Interkulturelle Bildung veranlasst damit strukturell die Aufnahme des Anderen und setzt über entsprechende Angebote die Konfrontation mit Fremdem und die Vorbereitung auf Fremde um. „Interkultur“ ist daher ein notwendiger Bezugsansatz, um kritisch-reflexive Auseinandersetzungen mit Rassismus und Diskriminierung zu ermöglichen und angebotsstrukturell zu verankern (Gieseke et al. 2005; Robak und Fleige 2012; Robak 2013). 
Darüber hinaus ist aber inzwischen mit einem Paradigma Transkultureller Bildung zu operieren. Dieser Ansatz ist zunächst von Welsch (2005) beschrieben worden und geht von kulturellen Verflechtungen aus, welche nicht nur Differenzen, sondern vor allem Gemeinsamkeiten produzieren. Transkulturelle Bildung konzeptualisiert über eine Fülle von Aneignungs- und Wissensformen Angebote für Bildung und Kompetenzentwicklung, die auf Bildungsbedürfnisse reagieren. Diese entstehen wiederum aufgrund sich modifizierender natio-ethno-kultureller (Zugehörigkeits-)Verhältnisse (Mecheril 2004), veränderter Wahrnehmungsformen und Bedeutungsproduktionen. Transkulturelle Bildung schließt an Interkulturelle Bildung an, bearbeitet darüber hinaus aber zur Erschließung der kulturellen Verflechtungen auch kommunikative und leiblich-emotionale Anteile von Bildungsprozessen und thematisiert Möglichkeiten von Wohlbefinden. Sie verfeinert im besonderen Maße die Wahrnehmungsund Empfindungsfähigkeit, fördert Humanität, Gerechtigkeit und die Entwicklung und Vergewisserung von Kulturalität (Göller 2000). Gesellschaftliche und kulturelle Hybridbildungsprozesse (Reckwitz 2006) bringen neue Herausforderungen für die Entfaltung von Kulturalität mit sich. Der Begriff der Transkulturellen Bildung adressiert auch Bildungsanforderungen im Horizont der Thematisierung und Bearbeitung von kulturellen Stereotypen, indem er neue gemeinsame Erfahrungen und darüber neue Deutungen evoziert, die sich auf andere Kulturen und die eigene Platzierung in diesen beziehen können. Transkultur geht von unabgeschlossenen Kulturen aus. Das Erlernen künstlerischer Praktiken und die interaktive Auseinandersetzung mit Themen der Beheimatung fördern die Ausformung von Mehrfachzugehörigkeiten. All dies kann als ein wichtiger Schlüssel für positive Verortung und den Wunsch für weitere Partizipation gesehen werden. Dann verändern sich Kulturen über die gemeinsame Gestaltung von Praktiken (vgl. Fleige et al. 2015, Kap. 7). Um einen Überblick über inter- und transkulturelle Angebotsstrukturen zu erhalten, bieten sich methodisch Programmanalysen an. Die hier beschriebenen Begriffe bilden die Folie für durchgeführte Programmanalysen, auf die im folgenden Kap. 4 Bezug genommen wird.

\section{Angebotsstrukturen für und mit Menschen mit Migrationsbiografien bzw. Geflüchtete im Spiegel von Programmanalysen}

Um zu analysieren, welche Integrationsvorstellungen in die Angebotsstrukturen eingelassen sind, können die Programme selbst herangezogen werden. Programmanalysen verweisen auf konzeptionelle Überlegungen; sie zeigen auf, welche Themen mit welchen Intentionen, Lernformen und Bildungsvorstellungen an wen gerichtet werden. ${ }^{9}$ Käpplinger (2016) untersucht in einer Programmanalyse die Volkshochschulangebote seit 1947 insbesondere unter dem Fokus, mit welchen Themen

\footnotetext{
9 Die Programmanalyse ist eine genuin erwachsenenpädagogische Forschungsmethode. Bei diesem Verfahren stehen die Programme als Untersuchungsgegenstand im Fokus (vgl. z. B. Käpplinger 2008; v. Hippel 2017). Grundlage der Auswertung sind Kodierpläne, die spezifisch kategorial entwickelt werden. Auf dieser Basis ist sowohl eine qualitative als auch quantitative Analyse möglich, indem die Ankündigungen jeweils einzeln betrachtet und ausgewertet werden.
} 
Angebote für Geflüchtete adressiert werden. Hier zeigt sich ein Höhepunkt in der politischen Thematisierung zwischen 1947 und 1950 und mit thematischen Schwerpunkten auf Flüchtlingsrecht und Fremdenfeindlichkeit in den Jahren 1989 bis 1997.

In 2012 wurde im Auftrag der Agentur für Erwachsenen- und Weiterbildung in Niedersachsen eine Programmanalyse zur Interkulturellen Bildung durchgeführt, um Einblicke in die Angebotsentwicklung dieser in der öffentlich geförderten Erwachsenenbildung in Niedersachsen zu erhalten. ${ }^{10}$ Dabei wurden zum einen Angebote nach bestimmten Kategorien quantitativ ausgezählt. Zum anderen ermöglicht die Betrachtung der Partizipationsportale eine Verschränkung mit einer qualitativen Perspektive, da die Portale auf der Grundlage einer inhaltsanalytischen Betrachtung der Ankündigungstexte identifiziert und anschließend in numerische Daten umgewandelt wurden.

Diese als Forschungsprojekt angelegte Programmanalyse untersuchte etwa $20 \%$ aller Angebote der öffentlich geförderten Erwachsenenbildung in Niedersachsen. Diese ergab für die Angebotsstrukturen der Interkulturellen und Transkulturellen Bildung insgesamt acht Partizipationsportale ${ }^{11}$ (vgl. Robak und Petter 2014). Die Analyse durch die Perspektive der Partizipationsportale eröffnet die Möglichkeit, die Zugangswege zu Bildung, Lernformen und Wissensformen (z. B. Portal 2: selbsttätig-kreativ o. Portal 4: verstehend-kommunikativ im transkulturellen Sinne) zu erfassen (Gieseke et al. 2005).

Es werden hier nur zentrale Ergebnisse genannt, die Aufschluss über Angebotsstrukturen für Menschen mit Migrationsbiografie bzw. Geflüchtete in Niedersachsen geben: An erster Stelle stehen mit ca. $40 \%$ des inter-/transkulturellen Bildungsangebots die Angebote speziell für Migrantinnen und Migranten (Portal 6), in der Regel sind dies Integrations- und Deutsch-als-Fremdsprache-Kurse (Sprachenbereich). Einen geringen Teil macht eine stadtteilorientierte und Personale Bildung aus. Der Zugang zu Interkultureller Bildung folgt hier der Prämisse der Integration und Inklusion, insbesondere durch Kulturtechniken wie Sprache und Praktiken sowie dem Aufbau von Netzwerken. Der Hauptteil der anstehenden Bildungsanstrengungen liegt demnach vor allem bei den Gruppen mit Migrationsbiografie. In allen anderen Portalen sind Menschen mit Migrationsbiografie bzw. Geflüchtete nicht explizit ausgewiesen. Dies überrascht für Geflüchtete nicht, da die Daten in 2012, also vor der letzten großen Migrationsbewegung, erhoben wurden. Aussagen über die Gruppenzusammensetzungen in den Seminaren all der anderen Portale sind auf dieser Grundlage nicht möglich. Ein transkultureller Bildungsansatz findet sich insgesamt nur in Ansätzen, er adressiert auch Menschen mit Migrationsbiografie, etwa mit

\footnotetext{
10 Für die Analyse wurden Programmhefte und zusätzliche Projektlisten der ausgewählten Einrichtungen mit einem Kodierplan ausgewertet, indem jedes einzelne Angebot und Projekt analysiert und kodiert werden. Dieser Kodierplan wurde eigens für diese Untersuchung erstellt und orientiert sich an bereits in der Forschung erprobten Kodierplänen (vgl. Schrader 2011; Gieseke und Opelt 2003). Neben den grundlegenden Daten wie Veranstaltungsform und Fachbereichszuordnung wurden auch die entwickelten Partizipationsportale zu Bildung und Kultur erfasst.

11 Die Bezeichnungen von Portal 6 und 8 beinhalten nicht wie die anderen Portale Hinweise auf querliegende Bearbeitungsformen, die aus Lern- und Aneignungsformen abgeleitet werden, sondern verweisen auf konkrete querliegende Zielgruppenadressierungen, die auszuweisen wichtig ist, um aktuelle Entwicklungen aufzuzeigen.
} 
einem Angebot über Transkulturalisierung infolge von Zweisprachigkeit, insgesamt aber mehr die Gesamtbevölkerung vor dem Hintergrund globaler Entwicklungen. Berücksichtigen wir das oben dargelegte Integrationskonzept, sind Angebote für Prozessebene I - Gestaltung der Lebenswelt über stadtteilbezogene Angebote - und Ebene III - mit einer Fokussierung auf Sprachangebote - erkennbar. Insgesamt wird eher ein Assimilationsbegriff sichtbar, der für die Gruppen mit Migrationsbiografie von einer Angleichung kultureller Differenz ausgeht, aber nicht alle Prozessebenen und Lerndimensionen einbezieht.

Um Entwicklungen in der inter- und transkulturellen Angebotsentwicklung nach 2015 im Zuge der letzten Migrationsbewegungen aufzuspüren, wurden in 2017 exemplarische Programmanalysen in drei Volkshochschulen und zwei Heimvolkshochschulen durchgeführt (zu den Gesamtergebnissen s. Robak 2017). ${ }^{12}$ Auch in 2017 dominieren, nach Fachbereichen betrachtet, die Sprachkurse bzw. Integrationskurse (51\% aller Angebote). Insbesondere die Zunahme an Angeboten im Bereich Alphabetisierung zeigt, dass auf die Geflüchteten reagiert wird, die Deutschland als Analphabeten erreichen. Die Zahlen in Portal 6 (Angebote speziell für Migrantinnen und Migranten) zeigen mit 35\% des interkulturellen Angebots, dass Menschen mit Migrationsbiografie nach wie vor die zentrale Zielgruppe sind, die sich integrieren soll. Die Ergebnisse zeigen aber insgesamt, dass sich die Angebote ausdifferenziert haben, d.h. die Mehrheitsbevölkerung wird breiter einbezogen. Zugenommen haben mit nun 25\% die Angebote im Bereich Kulturelle Bildung (2012: 19,4\%). Während eine Ausdifferenzierung dieses Angebotsspektrums festgestellt werden kann, das sich an alle richtet, konzentrieren sich Angebote an Geflüchtete auf Kochen und Tanz, aber auch Theater (Portal 2: selbsttätig-kreativ).

Angebote, die der Politischen Bildung zugeschrieben werden können, haben insgesamt zwar einen anteilsmäßigen Aufwuchs auf 12\% (2012: 4,5\%) und eine thematische Ausdifferenzierung zu verzeichnen, ${ }^{13}$ die Angebote richten sich jedoch überwiegend an die Mehrheitsgesellschaft und nicht explizit an die Geflüchteten. Es zeigt sich daran deutlich, dass nun die Mehrheitsgesellschaft adressiert wird, indem das interkulturelle Angebotsspektrum gesellschaftsrelevante Themen aufgreift, die aufgrund von Migration sichtbar werden: gesellschaftliche Spannungsfelder, Konflikte, die aus religiösen und politischen Überzeugungen heraus resultieren. Die Personale Bildung hingegen ist vom anteiligen Volumen her auf $7 \%$ zurückgegangen (2012: 12\%), weist aber im inhaltlichen Zuschnitt nun deutlicher interkulturelle Themen aus, die z.B. Ängste vor dem Islam, Fremdenfeindlichkeit und die Wirkungsweisen unterschiedlicher Deutungsmuster bezüglich Fremdheit und interkul-

\footnotetext{
12 Um Akzente und Entwicklungen in den Angebotsstrukturen zwischen 2012 und 2017 aufzuzeigen, werden Vergleiche vorgenommen, obwohl es sich nicht um die Programme derselben Einrichtungen handelt. Die Einrichtungen wurden jeweils von den Landesverbänden ausgewählt. Es handelt sich um Einrichtungen, die besonders aktiv in diesem Bereich sind.

13 Die von Zick und Klein (2014) aufgezeigten Dimensionen gruppenbezogener Menschenfeindlichkeit zeigen sich thematisch in den Angebotsstrukturen: z. B. Rassismus, Sexismus und die Befürwortung von Etabliertenvorrechten, Diskriminierung, Rechtsextremismus, Hass gegen Langzeitarbeitslose und Islamophobie. Es finden sich Angebote, die über Geschichte, Situation und Fluchtursachen in den Ländern, aus denen die Geflüchteten kommen, um Klischees und Vorurteile abzubauen. Auch die islamische Religion wird unter aufklärerischen Aspekten behandelt.
} 
tureller Differenz direkt thematisieren. Diese Angebote richten sich dabei zumeist an die Mehrheitsbevölkerung. Hier ein Beispiel:

„Seit dem Ausbruch der Revolution 2011 und dem darauf folgenden Bürgerkrieg sind mehr als 500.000 Syrer nach Deutschland geflohen. Sie alle möchten in Deutschland Fuß fassen und sollen gut integriert werden. Eine gewaltige Herausforderung, die auch Ängste in der deutschen Gesellschaft freigesetzt hat. Was macht den Deutschen Angst, worüber sorgen sich die Syrer? Fremde Umgangsformen, der Hang der Deutschen zu Verschlossenheit und Individualismus, unterschiedliche Vorstellungen von Moral und Liebe, Feministinnen mit Kopftuch, der deutsche Föderalismus und Sozialstaat - /Dozentin/beschreibt, mit welchen Kulturschocks Deutsche und Syrer im Umgang miteinander und im gegenseitigen Kennenlernen zu rechnen haben. "(VHS/Ort/)

Themen wie Fremdheit, Fremdsein, sich Beheimaten werden theaterpädagogisch und über die künstlerische Praxis des Theaterspielens bearbeitet. Neue transkulturelle Formen (Portal 4: verstehend-kommunikativ) finden sich etwa in der Domäne Theater. Diese Angebote richten sich explizit an Geflüchtete und an die Mehrheitsgesellschaft und laden zur gemeinsamen Gestaltung von Theaterstücken ein, etwa: „Fremd sein überwinden - Theaterseminar - Lebenswege“ (HVHS/Ort/). Angebote, die auch die Geflüchteten in partizipative Formen der Gestaltung demokratischer Teilhabe einbeziehen und der Politischen Bildung zugeordnet werden können, finden sich projektförmig im aushandelnd-reflexiven Portal, so etwa bei der gemeinsamen Gestaltung einer Zeitung (VHS/Ort/).

Auf die Prozessebenen bezogen wird deutlich, dass die Angebote, die explizit Geflüchtete adressieren, insbesondere auf Ebene III zielen, wobei ein Fokus auf Sprache liegt. Diese Angebote werden nun aber über selbsttätig-kreative Angebote der Kulturellen Bildung ausdifferenziert. Sie verfolgen projektförmig auch einen transkulturellen Zugang. Übergänge finden sich zur Prozessebene I, wenn es um die Gestaltung der Lebenswelt geht; es finden sich auch einige Angebote, die die individuellen Bildungskonstellationen berücksichtigen, etwa die Herkunft aus bildungsfernen Familien. Einzelne Angebote können Prozessebene II zugeordnet werden. Der Integrationsansatz hat sich deutlich ausdifferenziert und ermöglicht neben der Fokussierung auf Sprache Formen der Partizipation, Lebenswelt- und Kulturgestaltung, die die Geflüchteten einbeziehen. Auffälliger ist jedoch die breitere Adressierung der Mehrheitsgesellschaft. Es wird also ansatzweise ein Akkulturationsansatz deutlich, der auch Anpassungsthemen für die Mehrheitsgesellschaft nachbereitet. Das heißt, über Wissensvermittlung und Reflexion werden die gesellschaftlichen Folgewirkungen von Migration umfangreicher bearbeitet.

\section{Programm- und projektbezogene Ansätze der beruflichen Integration für Geflüchtete - eine exemplarische Analyse}

Ausgehend vom EU-finanzierten europäischen Projekt „Facilitating Migration and Workplace Integration with Qualified Personnel and Concepts“ (MaWIC) (Erasmus+), in dem ein Curriculum für ein Coaching-Konzept für die berufliche Integra- 
tion von Geflüchteten am Arbeitsplatz erarbeitet und umgesetzt wird, wurde für diesen Artikel eine ergänzende Recherche über Projekte durchgeführt, die sich in 2017 der beruflichen Integration Geflüchteter widmen. Die leitende Frage ist: In welcher Form wird die Integration Geflüchteter auf Prozessebene II - Professionalisierung und Qualifizierung konzeptualisiert? Die Internetrecherche wurde im Schwerpunkt in Niedersachsen und Nordrhein-Westfalen durchgeführt. Insgesamt wurden 36 Projekte identifiziert, die sich an Geflüchtete richten. Unter den analysierten Projekten befinden sich mehr Projekte mit dem Ziel, die Teilnehmenden in eine feste Anstellung zu vermitteln (23 Nennungen) als Maßnahmen, die qualifizieren, aber nicht direkt in eine Anstellung führen (16 Nennungen). Die am häufigsten angegebenen Instrumente sind arbeitsplatzbezogene Lernformen wie Praktika, Internships, Hospitationsplätze, Coaching, Mentoring und Patenmodelle mit dem Ziel der direkten Anstellung (26 Nennungen). Auffällig ist mit zwölf Nennungen die Fokussierung auf die Vermittlung berufsbezogener Sprachkenntnisse und Sprachförderung allgemein. Projekte bereiten durch Informationen und Berufsvorbereitungsmaßnahmen auf den Arbeitsmarkt vor (9 Nennungen). Es finden sich auch Maßnahmen, die die Betriebe darauf vorbereiten, Geflüchtete einzustellen, etwa durch Beratung, interkulturelles Training und gezielte Hilfestellungen.

Es werden überwiegend Projekte konzipiert und durchgeführt, die sich nicht auf spezifische Herkunftsländer der Geflüchteten beziehen, bei 9 Projekten finden sich konkrete spezifische Angaben, die Geflüchtete aus dem Nahen Osten (Syrien und Irak), dem Mittleren Osten (Afghanistan und Pakistan) sowie aus Nordafrika (Marokko, Niger und Eritrea) adressieren. Die Projekte wurden überwiegend neu geschaffen, es wurden aber auch bestehende Projekte und Maßnahmen erweitert.

Die Zielsetzungen, Zeitformate und die Lernformen verweisen darauf, dass berufliche Integration, die in Kooperation mit Unternehmen stattfindet, konkret an der Einübung am Arbeitsplatz ansetzt, um so die Geflüchteten unmittelbar mit den Tätigkeiten und Arbeitskulturen vertraut zu machen. Damit folgen die Unternehmen ihrem Bedürfnis, interkulturelle Unterschiede, Unterschiede in den Arbeitskulturen und Bildungsvoraussetzungen aufzufangen und eine employability zu prüfen. Es wird nicht unmittelbar eingestellt. Die identifizierten Maßnahmen zielen auf eine konkrete Integration in die Unternehmen und eine Vorbereitung darauf. Dies setzt voraus, dass Kenntnisse, Fähigkeiten und auch Abschlüsse vorhanden sind, an die angeschlossen werden kann.

Insgesamt kann festgehalten werden, dass sich Unternehmen gemeinsam mit Bund, Land und vor allem EU-Finanzierungen der beruflichen Integration widmen, gleichwohl keine Aussage über den bundesweiten Deckungsgrad getroffen werden kann.

\section{Ausblick}

Für die Entwicklung von Angebotsstrukturen kann auf vielfältige Erfahrungen der Angebotsentwicklung für Menschen mit Migrationsbiografie aus verschiedenen Phasen zurückgegriffen werden. Ein Schwerpunkt der Integration kann durch Sprache identifiziert werden. Die Angebotsstrukturen wurden in den vergangenen drei Jahren 
ausdifferenziert und entwickeln sich auf allen drei Prozessebenen. Eine transkulturelle Haltung und Bildung findet sich erst in Ansätzen; der Integrationsbegriff ist stark assimilativ geprägt und bezieht sich deutlicher auf interkulturelle Bildungsgedanken. Innovative Ansätze entstammen der Kulturellen Bildung und finden sich etwa selbsttätig-kreativ für Prozessebene III. Auch die Ansätze der beruflichen Integration haben sich ausdifferenziert; Prozessebene II wird besonders auf der Grundlage von Mischfinanzierungen, unterstützt durch die Betriebe als arbeitsplatzbezogene Lernformen offeriert. Auch wenn kein Gesamtkonzept der Integration durch Bildung erkennbar ist, gibt es Angebote auf allen drei Prozessebenen, die aber für sich vereinzelt sind. Demnach ist es notwendig, einen integrativen Ansatz auf der Grundlage der Prozessebenen und Lerndimensionen zu entwickeln.

Open Access Dieser Artikel wird unter der Creative Commons Namensnennung 4.0 International Lizenz (http://creativecommons.org/licenses/by/4.0/deed.de) veröffentlicht, welche die Nutzung, Vervielfältigung, Bearbeitung, Verbreitung und Wiedergabe in jeglichem Medium und Format erlaubt, sofern Sie den/die ursprünglichen Autor(en) und die Quelle ordnungsgemäß nennen, einen Link zur Creative Commons Lizenz beifügen und angeben, ob Änderungen vorgenommen wurden.

\section{Literatur}

Alheit, P. (1995). „Biographizität“ als Lernpotential. Konzeptionelle Überlegungen zum biographischen Ansatz in der Erwachsenenbildung. In H.-H. Krüger \& W. Marotzki (Hrsg.), Erziehungswissenschaftliche Biographieforschung (S. 276-307). Opladen: Leske + Budrich.

Alheit, P., \& Dausien, B. (2002). Bildungsprozesse über die Lebensspanne und lebenslanges Lernen. In R. Tippelt (Hrsg.), Handbuch Bildungsforschung (S. 565-585). Wiesbaden: Springer.

Arnold, R. (1985). Deutungsmuster und pädagogisches Handeln in der Erwachsenenbildung: Aspekte einer Sozialpsychologie der Erwachsenenbildung und einer erwachsenenpädagogischen Handlungstheorie. Bad Heilbrunn: Klinkhardt.

Bade, K. (2017). Migration - Flucht - Integration. Kritische Politikbegleitung von der „Gastarbeiterfrage“ bis zur „Flüchtlingskrise“. Erinnerungen und Beiträge. Karlsruhe: Loeper.

Baethge, M., \& Baethge-Kinsky, V. (2004). Der ungleiche Kampf um das lebenslange Lernen: Eine Repräsentativ-Studie zum Lernbewusstsein und -verhalten der deutschen Bevölkerung. In M. Baethge \& V. Baethge-Kinsky (Hrsg.), Der ungleiche Kampf um das lebenslange Lernen (S. 11-200). Münster: Waxmann.

BAMF - Bundesamt für Migration und Flüchtlinge (2015). Bericht zur Integrationskursgeschäftsstatistik für das Jahr 2015. Nürnberg. http://www.bamf.de/SharedDocs/Anlagen/DE/Downloads/ Infothek/Statistik/Integration/2015/2015-integrationskursgeschaeftsstatistik-gesamt_bund.pdf? blob=publicationFile. Zugegriffen: 11. Juli 2018

BAMF - Bundesamt für Migration und Flüchtlinge (2018). IAB-BAMF-SOEP-Befragung von Geflüchteten 2016. Nürnberg. https://www.bamf.de/SharedDocs/Anlagen/DE/Publikationen/Forschungs berichte/fb30-iab-bamf-soep-befragung-gefluechtete-2016.pdf?_blob=publicationFile. Zugegriffen: 16. Juli 2018

Berry, J. W. (2005). Living succesfully in two cultures. International Journal of Intercultural Relations, $29,697-712$.

Bhabha, H. K. (2000). Die Verortung der Kultur. Tübingen: Stauffenburg.

BMBF - Bundesministerium für Bildung und Forschung (2018). Erste Ergebnisse aus Studie „Geflüchtete Familien“. Untersuchung liefert repräsentative Erkenntnisse über Bildungshintergrund von Flüchtlingen / Wanka: „Daten sind auch für Migrationsforschung wertvoll“. Berlin. https://www.bmbf.de/de/ erste-ergebnisse-aus-studie-gefluechtete-familien-4698.html. Zugegriffen: 21. Apr. 2018.

Böhmer, A. (2016). Bildung als Integrationstechnologie? Neue Konzepte für die Bildungsarbeit mit Geflüchteten. Bielefeld: transcript.

Boos-Nünning, U. \& Karakaşoğlu, Y. (2005). Familialismus und Individualismus. Zur Bedeutung der Familie in der Erziehung von Mädchen mit Migrationshintergrund. In U. Fuhrer \& H.-H. Uslu- 
can (Hrsg.), Familie, Akkulturation und Erziehung. Migration zwischen Eigen- und Fremdkultur (S. 126-149). Stuttgart: Kohlhammer.

bpb - Bundeszentrale für Politische Bildung (2017). Zahlen zu Asyl in Deutschland: Infografiken nach Daten des Bundesamtes für Migration und Flüchtlinge. Berlin. https://www.bpb.de/gesellschaft/ migration/flucht/218788/zahlen-zu-asyl-in-deutschland. Zugegriffen: 21. Apr. 2018.

Brandt, P., Loreit, F., \& Schöll, I. (2016). Wir müssen uns die Sache ein bisschen schwerer machen. DIE Zeitschrift im Gespräch mit Ingrid Schöll zu Wertefragen der Weiterbildung im Zuge aktueller Integrationsaufgaben. DIE Zeitschrift für Erwachsenenbildung, 2, 24-27.

Dehnbostel, P. (2008). Berufliche Weiterbildung. Grundlagen aus arbeitnehmerorientierter Sicht. Berlin: edition sigma.

Dybowski, G., \& Thomssen, W. (1976). Praxis und Weiterbildung. Berlin: unveröff. Manuskript.

Esser, H. (2001). Integration und ethnische Schichtung. Arbeitspapier Nr. 40. Mannheim: Mannheimer Zentrum für Europäische Sozialforschung.

Fleige, M., Gieseke, W., \& Robak, S. (2015). Kulturelle Erwachsenenbildung. Strukturen - Partizipationsformen - Domänen. Bielefeld: wbv.

Friebel, H. (2008). Die Kinder der Bildungsexpansion und das „Lebenslange Lernen“. Augsburg: Ziel.

Friebel, H., Epskamp, H., Knobloch, B., Montag, S., \& Toth, S. (2000). Bildungsbeteiligung: Chancen und Risiken: eine Längsschnittstudie über Bildungs- und Weiterbildungskarrieren in der „Moderne“. Opladen: Springer.

Gieseke, W. (2008). Bedarfsorientierte Angebotsplanung in der Erwachsenenbildung. Bielefeld: wbv.

Gieseke, W. (2010). Erfahrungsorientierung - Erfahrung. In R. Arnold, S. Nolda \& E. Nuissl (Hrsg.), Wörterbuch Erwachsenenbildung (2. Aufl. S. 76-78). Bad Heilbrunn: UTB.

Gieseke, W. (2016). Lebenslanges Lernen und Emotionen. Wirkungen von Emotionen auf Bildungsprozesse aus beziehungstheoretischer Perspektive. Bielefeld: wbv.

Gieseke, W., \& Opelt, K. (2003). Erwachsenenbildung in politischen Umbrüchen. Programmforschung Volkshochschule Dresden 1945-1997. Opladen: Leske + Budrich.

Gieseke, W., Opelt, K., Stock, H., \& Borjesson, I. (2005). Kulturelle Erwachsenenbildung in Deutschland: Exemplarische Analyse Berlin/Brandenburg. Münster: Waxmann.

Göller, T. (2000). Kulturverstehen, Grundprobleme einer epistemologischen Theorie der Kulturalität und kulturellen Erkenntnis. Würzburg: Königshausen und Neumann.

Griese, H. (1984). Der gläserne Fremde. Bilanz und Kritik der Gastarbeiterforschung und der Ausländerpädagogik. Leverkusen: Leske + Budrich.

Hamburger, F., Badavia, T., \& Hummrich, M. (2005). Migration und Bildung. Über das Verhältnis von Anerkennung und Zumutung in der Einwanderungsgesellschaft. Wiesbaden: VS.

Hauenschild, K., Robak, S., \& Sievers, I. (2013). Diversity Education: Zugänge - Perspektiven - Beispiele. Frankfurt a.M.: Brandes \& Apsel.

Heckmann, F. (2015). Integration von Migranten. Einwanderung und neue Nationenbildung. Wiesbaden: Springer.

Heinemann, A. (2014). Teilnahme an Weiterbildung in der Migrationsgesellschaft: Perspektiven deutscher Frauen mit „Migrationshintergrund“. Bielefeld: transcript.

Heinemann, A., \& Robak, S. (2012). Interkulturelle Erwachsenenbildung. In Enzyklopädie Erziehungswissenschaft Online (S. 1-54). ISSN 2191-8325.

Hessischer Volkshochschulverband e. V. (2016). Integration. Hessische Blätter für Volksbildung, Bd. 4.

v. Hippel, A. (2017). Theoretische Perspektiven auf Programmplanung in der Erwachsenenbildung. Zeitschrift für Weiterbildungsforschung - Report, 40(2), 199-209. https://doi.org/10.1007/s40955-0170090-4. Zugegriffen: 11. Juli 2018.

Hofheinz, M. (2016). Religion: Motor oder Hemmschuh der Integration? Oder: Mehr Religionsfreiheit wagen. Hessische Blätter für Volksbildung, Bd. 4 (S. 345-352).

Käpplinger, B. (2008). Programmanalysen und ihre Bedeutung für Pädagogische Forschung. Forum Qualitative Sozialforschung, 37 (1). www.qualitative-research.net/index.php/fqs/article/view/333/728. Zugegriffen: 11. Juli 2018.

Käpplinger, B. (2016). Generations of adult education programs - lost and found imaginations. 8th ESREA Triennial Research Conference at Maynooth University.

Kaschuba, W. (1995). Kulturen - Identitäten - Diskurse. Berlin: Akademie.

King, V., \& Koller, H.C. (2006). Adoleszenz-Migration - Bildung. Bildungsprozesse Jugendlicher und junger Erwachsener mit Migrationshintergrund. Wiesbaden: VS.

Koop, J., \& Schäfers, B. (2006). Grundbegriffe der Soziologie (10. Aufl.). Wiesbaden: Springer.

Kraus, K. (2006). Vom Beruf zur Employability? Zur Theorie einer Pädagogik des Erwerbs. Wiesbaden: Springer VS. 
Krause, D., Zick, A., \& Küpper, B. (Hrsg.). (2016). Gespaltene Mitte - Feindselige Zustände: Rechtsextreme Einstellungen in Deutschland 2016. Bonn: J. H. W. Dietz Nachf..

Mecheril, P. (2003). Prekäre Verhältnisse. Über natio-ethno-kulturelle (Mehrfach)Zugehörigkeit. Münster: Waxmann.

Mecheril, P. (2004). Einführung in die Migrationspädagogik. Weinheim: Beltz.

Mecheril, P., Castro Varela, M. M., Dirim, İ., Kalpaka, A., \& Melter, C. (2010). Bachelor/Master: Migrationspädagogik. Weinheim: Beltz.

Öztürk, H. (2012). Soziokulturelle Determinanten der beruflichen Weiterbildungsbeteiligung von Erwachsenen mit Migrationshintergrund in Deutschland. Zeitschrift für Weiterbildungsforschung - Report, 35(4), 21-32.

Öztürk, H. (2014). Migration und Erwachsenenbildung. Bielefeld: wbv.

Öztürk, H. (2016). Diversitätsbewusste Weiterbildung im Migrationskontext: Anforderungen an das Zusammenspiel von Weiterbildungsforschung, -praxis und -politik. Hessische Blätter für Volksbildung, 4, 324-333.

Pielage, P., Pries, L., \& Schultze, G. (2012). Soziale Ungleichheit in der Einwanderungsgesellschaft. Kategorien, Konzepte, Einflussfaktoren. (Tagungsdokumentation im Auftrag der Abteilung Wirschaftsund Sozialpolitik der Friedrich-Ebert-Stiftung). http://library.fes.de/pdf-files/wiso/09198.pdf. Zugegriffen: 12. Apr. 2018.

Reckwitz, A. (2003). Grundelemente einer Theorie sozialer Praktiken. Eine sozialtheoretische Perspektive. Zeitschrift für Soziologie, 32(4), 282-301.

Reckwitz, A. (2006). Das hybride Subjekt: Eine Theorie der Subjektkulturen von der bürgerlichen Moderne zur Postmoderne. Weilerswist: Velbrück Wissenschaft.

Reichertz, J. (2003). Die Abduktion in der qualitativen Sozialforschung. Wiesbaden: VS.

Robak, S. (2012). Kulturelle Formationen des Lernens. Zum Lernen deutscher Expatriates in kulturdifferenten Arbeitskontexten in China - die versäumte Weiterbildung. Münster: Waxmann. Habilitationsschrift

Robak, S. (2013). Interkultur - Transkultur - Hybdridkultur. Spannungsfelder und (weiter)bildungsrelevante Implikationen. Hessische Blätter für die Volksbildung, 1, 14-28.

Robak, S. (2015). Angebotsentwicklung für Flüchtlinge. Rahmenbedingungen und Strukturen der Bildungsarbeit mit Asylsuchenden. Erwachsenenbildung. Vierteljahresschrift für Theorie und Praxis, 4, $10-13$.

Robak, S. (2016). Zur Konzeptualisierung von Diversität in der Erwachsenenbildung und Weiterbildung. Begriffsentwicklung und empirische Ergebnisse aus den Bereichen Inter-/Transkultureller und Kultureller Bildung. In K. Dollhausen \& S. Muders (Hrsg.), Diversität und Lebenslanges Lernen. Aufgaben für die organisierte Weiterbildung (S. 27-45). Bielefeld: wbv.

Robak, S. (2017). Entwicklungen und Akzente in der Inter- und Transkulturellen Bildung. Der verstehend-kommunikative Zugang kultureller Bildungsangebote. Hessische Blätter für Volksbildung, 4, 333-351.

Robak, S., \& Fleige, M. (2012). Tendenzen in Angebotsstrukturen kultureller und interkultureller Bildung. Der pädagogische Blick, 20(4), 233-248.

Robak, S., \& Grawan, F. (2016). Inter- und Transkulturelle Bildung mit Fokus auf Mehrfachdiskriminierung und Mehrfachzugehörigkeit. Hessische Blätter für Volksbildung, 4, 353-363.

Robak, S., \& Petter, I. (2014). Programmanalyse zur interkulturellen Bildung in Niedersachsen. Bielefeld: wbv.

Schäffter, O. (1991). Modi des Fremderlebens. Deutungsmuster im Umgang mit Fremdheit. In O. Schäffter (Hrsg.), Das Fremde. Erfahrungsmöglichkeiten zwischen Faszination und Bedrohung (S. 11-42). Wiesbaden: Springer.

Scharfe, M. (2002). Menschenwerk. Erkundungen über Kultur. Köln: Böhlau.

Schiersmann, C. (2006). Profile lebenslangen Lernens. Weiterbildungserfahrungen und Lernbereitschaft der Erwerbsbevölkerung. Bielefeld: wbv.

Schiffauer, W., Eilert, A., \& Rudolff, M. (2017). So schaffen wir das. Eine Zivilgesellschaft im Aufbruch. 90 wegweisende Projekte mit Geflüchteten. Bielefeld: transcript.

Schrader, J. (2011). Struktur und Wandel der Weiterbildung. Bielefeld: wbv.

Schüssler, I. (2000). Zu Notwendigkeiten und Voraussetzungen nachhaltigen Lernens in der Erwachsenenbildung - Thesen zum notwendigen Wandel von Lernkulturen. In M. Zöller (Hrsg.), Vom Betreuungsstaat zur Bürgergesellschaft - Kann die Gesellschaft sich selbst regeln und erneuern (S. 200-208). Köln: Wirtschaftsverlag.

Sievers, I., \& Grawan, F. (2018). Fluchtmigration, gesellschaftliche Teilhabe und Bildung: Handlungsfelder und Erfahrungen. Frankfurt a. M.: Brandes \& Aspel. 
Sievers, I., Griese, H., \& Schulte, R. (2010). Bildungserfolgreiche Transmigranten. Eine Studie über deutsch-türkische Migrationsbiographien. Frankfurt a.M.: Brandes \& Apsel.

Sprung, A. (2009). Migration, Rassismus, Interkulturalität - (k)ein Thema für die Weiterbildungsforschung? Magazin Erwachsenenbildung.at. Das Fachmedium für Forschung, Praxis und Diskurs, 7(8), $2-10$.

Welsch, W. (2005). Auf dem Weg zu transkulturellen Gesellschaften. In L. Allolio-Näcke, B. Kalscheuer \& A. Manzeschke (Hrsg.), Differenzen anders denken. Bausteine zu einer Kulturtheorie der Transdifferenz (S. 314-341). Frankfurt a.M.: Campus.

Zick, A., \& Klein, A. (2014). Fragile Mitte - feindselige Zustände: Rechtsextreme Einstellungen in Deutschland. Bonn: Friedrich-Ebert-Stiftung.

Ziese, M., \& Gritschke, C. (2016). Geflüchtete und Kulturelle Bildung. Formate und Konzepte für ein neues Praxisfeld. Bielefeld: transcript.

Zimmer, V. (2013). Anspruch und Wirklichkeit von Integrationskursen im Lichte erwachsenenpädagogischer Forschung. www.die-bonn.de/doks/2013-inklusion-01.pdf. Zugegriffen: 21. Apr. 2016.

Zimmer, V., Fleige, M., Lücker, L., \& Thom, S. (2015). Expertise „Diversität und Weiterbildung“ im Auftrag von Lernen vor Ort Bremen/Bremerhaven. http://docplayer.org/17706247-Diversitaet-undweiterbildung-in-bremen-und-bremerhaven.html. Zugegriffen: 18. Sept. 2015.

Zimmermann, D. (2012). Migration und Trauma. Pädagogisches Verstehen und Handeln in der Arbeit mit jungen Flüchtlingen. Gießen: Psychosozial-Verlag. 